\title{
Analysis of terahertz systems and analytical methods for modeling their optical efficiency.
}

\author{
S. Kehoe, N. Trappe, J. A. Murphy, Member, IEEE.
}

\begin{abstract}
This research includes theoretical analysis and experimental testing of terahertz systems operating in the wband range. In modelling these long wavelength systems we use Gaussian Beam mode analysis, a novel technique which can be efficiently used to predict optical behaviour of systems that borrow components from both traditionally established optical and radio receiver techniques. This analytical technique neatly includes diffraction, which dominates propagation in this wavelength range. We are currently investigating the most numerically efficient and stable ways of utilising these modes in optical analysis and present our findings. In parallel, in an experimental campaign, we are characterising standing waves or multiple reflections which occur within terahertz systems. We present the experimental arrangement and outline potential methods to reduce the influence of multiple reflections in terahertz systems.
\end{abstract}

Index Terms-Gaussian Beam Mode Analysis, quasioptical systems, standing waves, sub-millimetre radiation

\section{INTRODUCTION}

$\mathrm{M}$ ETHODS for modelling the behaviour of Quasioptical systems based on the principles of Gaussian Beam Mode Analysis[1] are now widely documented and used as standard methods for modelling systems involving terahertz frequency radiation. In the first part of this paper we shall study two analytical techniques used in Gaussian Beam Mode analysis and their stability when subjected to increasing computational intensity. The two different techniques investigated are the use of Hermite-Gaussian relations and also singular value decomposition.

The second part of this paper concerns an experimental campaign investigating the effects of standing waves on systems operating at sub-millimetre wavelengths. Gaussian Beam mode analysis has been utilized in modelling the power coupled between two horns separated by a known distance [2]. When this separation distance is changed the power coupled between the two horns varies periodically as expected but also illustrates more anharmonic behaviour associated with high Q cavities. We investigate and validate models to simulate this effect and also investigate techniques to reduce the magnitude of standing waves using apertures and blockages. A first order model of the multiple reflections is also analyzed in order to

S. Kehoe, N. Trappe and J. A. Murphy are with the Experimental Physics Dept., National University of Ireland Maynooth, Ireland. establish reflection coefficients from antennas and optical components commonly utilized in quasioptical systems. This standing wave behaviour is the subject of the second study briefly outlined in this paper.

\section{NUMERICAL EFFICIENCY OF METHODS OF GAUSSIAN BEAM MODE ANALYSIS}

\section{A. Theory and Introduction}

Gaussian Beam mode analysis is a powerful analytical technique in modelling the behaviour of quasioptical systems, practically it is simply the breaking down of a complex beam profile into more manageable parts which may be propagated accurately through systems of lenses and mirrors more easily than the whole beam profile. Once the individual modes have undergone any transformation that represents the system through which the beam is travelling, they are finally reintegrated into a second beam profile using the following equation:

$$
\sum_{m} A_{m} \psi_{m},
$$

where $A_{m}$ is the mode coefficient and $\psi_{m}$ is a complex function describing the mode.

Two different methods were used to reconstruct the chosen beam profiles. First the use of Hermite-Gaussian [1] relations, from this point on referred to as HG relations, was tested. These HG relations use a set of recursion relations to construct the mode coefficients before and after propagation through a system.

The second method used was based on singular value decomposition, from this point on referred to as SVD method, a matrix method that allows one to find the pseudo-inverse of non-square matrices. This method allows us to treat a set of mode coefficients and a set of mode functions as two matrices allowing us to quickly find a set of post-propagation mode coefficients without having to resort to more computationally intensive recursion methods.

\section{B. HG Relations Method}

Using the method described in [1] a set of mode coefficients $A_{m}$ were created for the beam profile; the profile was then reconstructed close to the aperture plane using increasing numbers of modes in the reconstruction. With increasing numbers of modes the reconstruction more closely 
matched the original beam profile, however once around 180 modes and 90 modes, for a top-hat field reconstruction and Gaussian field respectively, was reached the method became unstable and broke down $c f$. figure 1 .
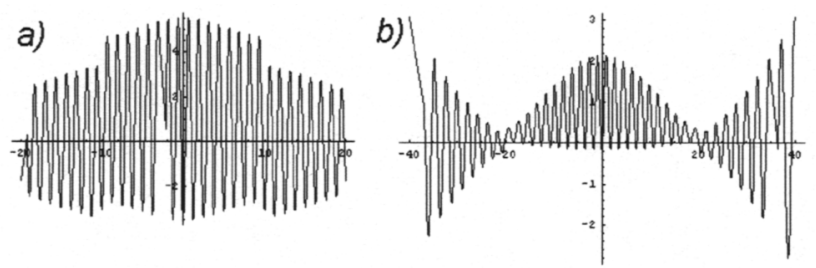

Fig. 1 a) Reconstruction of a Top Hat Field using 180 modes. b) Reconstruction of a Gaussian Field at 90 modes. Both plots showing the breakdown of the HG relation method.

\section{SVD Method}

The SVD method proved to be much more stable. This second technique uses two matrices; $\mathbf{E}_{\mathbf{m}}$, the matrix of electric fields for each mode $m$, and the pseudo inverse of $\Psi_{\mathbf{n m}}$, the matrix of modes, where $n$ is the range over which you wish to view the profile of the beam.

Again reconstructions of the beam were carried out for increasing numbers of modes until the point at which the method became unstable for the Gaussian profile (270 modes), and also the point at which the reconstruction of the top-hat beam took an unfeasibly long time to be calculated, (330 modes) in approximately 50 minutes, $c f$. figure 2 .

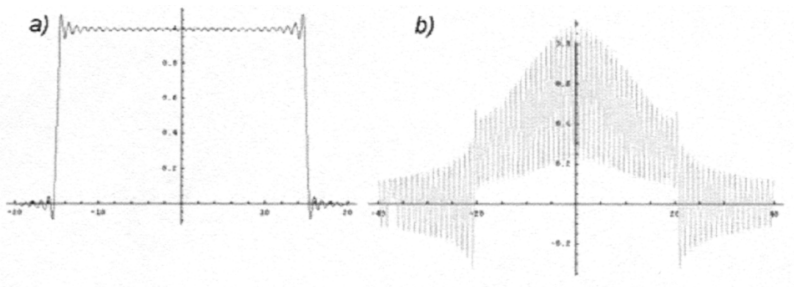

Fig. 2 a) Reconstruction of a Top Hat Field at 330 modes, 50 minutes computational time b) Reconstruction of a Gaussian Field at 270 modes, showing instability at high number of modes.

While this method is computationally intensive, with computation times increasing greatly over that of HG methods the overall stability of the method is apparent in methods ability to handle higher numbers of modes.

\section{Modelling And Understanding Standing Waves}

\section{A. Theory and Introduction}

Standing waves pose a considerable problem in $\mathrm{THz}$ systems; especially in areas such as $\mathrm{THz}$ astronomy where heterodyne receivers are used in $\mathrm{THz}$ medical and security imaging where multiple reflections reduce and distort the image quality. To this end modelling of standing wave systems and experimental investigation into the nature of standing waves at sub-millimetre wavelengths is essential.

\section{B. Example of Experiment}

The following is an example of one of the experiments carried out in the course of this research. The system was set up as described in figure 3, with the source and receiving horns facing each other. The source horn was moved away from the receiving horn using the moveable mount and the power being coupled was measured every $0.05 \mathrm{~mm}$. A plot of the coupled power versus the separation distance was normalized and is plotted below in figure 4 .

Fig. 3 Photograph of experimental setup used in investigating standing waves.

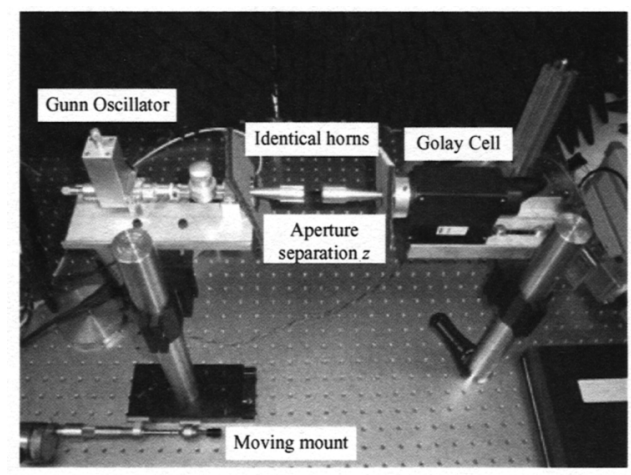

\section{Experimental Results}

Preliminary results for the above experimental setup have been encouraging to validate a simple approximate model. The normalized set of results from the experiment have been compared to two different developed models, an analytical solution modelling the standing waves in the above system and a model developed from HG relation method described earlier $c f .4$

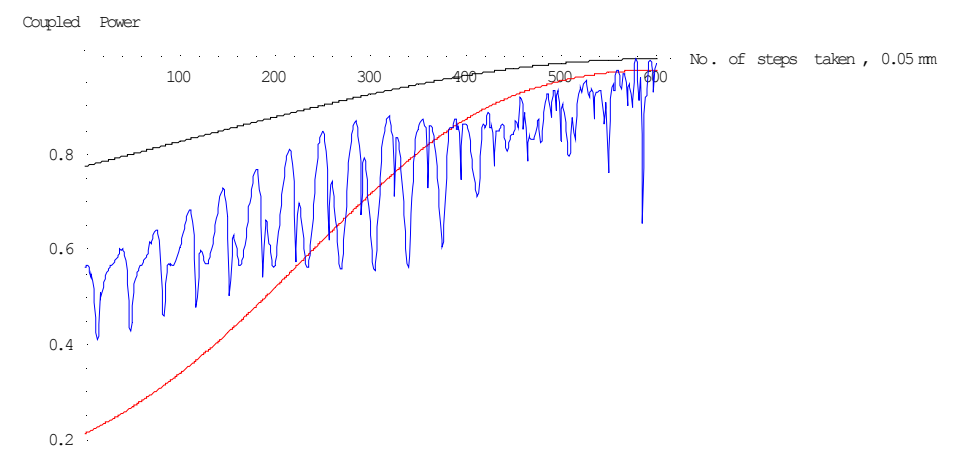

Fig. 4 Comparison of experimental results (blue), model developed using Mathematica (red), and the analytical solution from Goldsmith [3](black)

[1] J. A. Murphy, A. Egan, "Examples of Fresnel diffraction using Gaussian modes." Eur. J. Phys .14 (1993)

[2] N. Trappe, J. A. Murphy, "Gaussian Beam Mode Analysis of Standing Waves Between Two Coupled Corrugated Horns." IEEE Trans. Antenna and Propagation, Vol.53 No. 52005

[3] P. F. Goldsmith, Quasioptical Systems, Gaussian Beam Quasioptical Propagation and Applications, IEEE Press/Chapman \& Hall, 1998 